Dr. med.

Ulrich

Mutschler,

Hildesheim

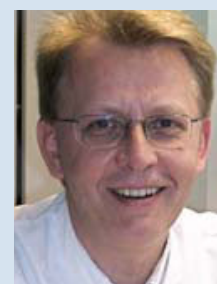

Prof. Dr

med.

Kurt Rasche,

Wuppertal

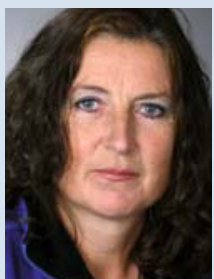

Dr. med.

Kirsten

Stollhoff,

Hamburg

\title{
Kinder und Jugendliche schlafen wieder weniger
}

\section{Wissenschaftler verglichen noch einmal Daten aus dem Jahr 1907 mit denen eigener Ergebnisse zur Schlafdauer von deutschen Kindern und Jugendlichen aus den Jahren 2006 und 2008 und stellten auch einen Vergleich mit Schlafzeiten aus den 1970er-Jahren her.}

D ie Schlafdauer soll sich während der letzten 100 Jahre sowohl bei Erwachsenen wie auch bei Heranwachsenden verkürzt haben. Die Datenlage ist aber inkohärent. In der Altersgruppe der 10- bis 15-Jährigen fand man z. B. im Zeitraum von 1985 bis 2004 eine Reduktion der durchschnittlichen Schlafdauer um ca. eine halbe Stunde [Acta Paediatr 2007; 96 1011-4]. Andere Studien belegten eine Reduktion der Schlafdauer von Kindern zwischen 1910 und 1963 um ca. 1,5 Stunden [J Educ Psychol 1913; 4: 138-47, Bull Psychon Soc 1975; 6: 47-8]. Allerdings sind diese Daten fragwürdig, da die Autoren der Arbeit aus dem Jahr 1913 ihr Untersuchungskollektiv als „exzessiv müde“ beschrieben. Darüber hinaus bestanden erhebliche Unterschiede der Schlafzeiten zu einer anderen Arbeit aus derselben Zeit, deren Daten plausibler erschienen [5].

Die Aufstehzeiten unterschieden sich zwischen ca. 1907 und ca. 2007 nicht signifikant. So war die mittlere Aufstehzeit bei den 11- bis 12-Jährigen im Jahr 1907 6.35 Uhr und im Jahr 20076.26 Uhr (kein signifikanter Unterschied).

Der Zeitpunkt des Zubettgehens war dagegen vor 100 Jahren später, sodass die Jugendlichen im Jahr 2007 ca. eine halbe Stunde länger schliefen als 1907. Beispielsweise gingen die 11- bis 12-Jährigen
1907 um 21.45 Uhr, 2007 aber schon um 20.54 Uhr zu Bett ( $<0,001)$. In derselben Altersgruppe betrug die Schlaflänge 19078 h 55 min, 2007 dagegen 9 h 31 min ( $<<0,001)$. Im Vergleich zu Daten aus den 1970er-Jahren schliefen Kinder aus der genannten Altersgruppe damals aber länger als 1907 oder 2007, und zwar fast zehn Stunden [Sleep 1998; 11: 378-86].

Das Ergebnis der Wissenschaftler: Kinder und Jugendliche schlafen heute länger als vor 100 Jahren, aber kürzer als vor 30 Jahren.

Kommentar: Diese Ergebnisse sind trotz des methodisch problematischen Vergleichs von Daten aus unterschiedlichen Studien zu sehr unterschiedlichen Zeitpunkten sehr interessant. Das Vorurteil, dass Kinder heute kürzer schlafen als vor 100 Jahren, kann aber aufgrund der Datenlage weitgehend widerlegt werden. Kinder schlafen heute ca. eine halbe Stunde länger als zu Beginn des letzten Jahrhunderts. Dies liegt wahrscheinlich

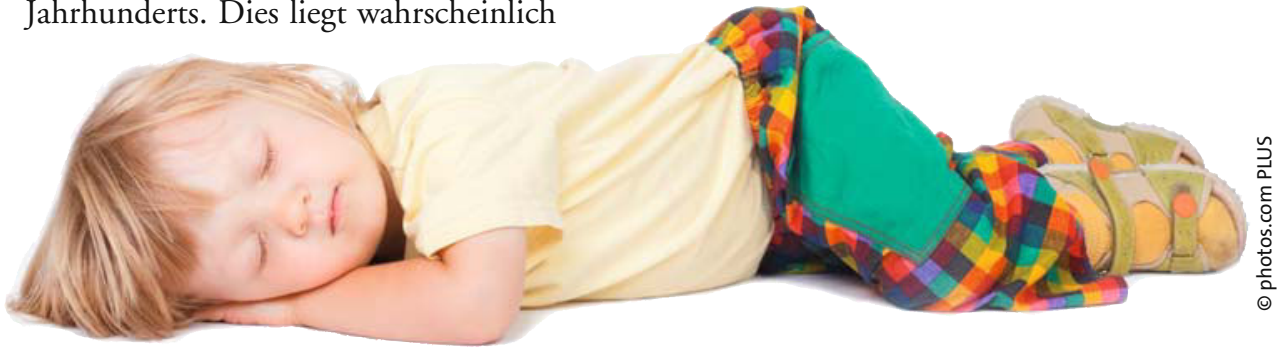

daran, dass sie damals intensiver in andere häusliche Aufgaben, ja sogar in die Arbeit der Erwachsenen integriert wurden und somit erst später zu Bett gehen konnten. Diese Interpretation kann damit begründet werden, dass die Untersuchung aus dem Jahr 1907 überwiegend bei Kindern der Unter- und Mittelschicht, 2007 aber eher bei Kindern, die zumindest aus der Mittelschicht stammten, durchgeführt wurde.

Interessant ist außerdem, dass Kinder heute weniger schlafen als noch vor 30 Jahren, was möglicherweise auf eine stärkere äußere Ablenkung durch die auch in den Abend- und Nachtstunden zur Verfügung stehenden Medien (Fernsehen, Internet usw.) zurückgeführt werden kann. Ob dies dazu führen sollte, z. B. den Beginn der Schule auf einen späteren Zeitpunkt zu verlagern, muss allerdings angezweifelt werden. Vielmehr sollten Kinder und Jugendliche aus schlafmedizinischer Sicht schon frühzeitig die Regeln einer adäquaten Schlafhygiene verinnerlichen.

Prof. Dr. Kurt Rasche

Randler C. Sleep length in German children and adolescents. Comparing 1907 with 2006-2008. Somnologie 2009;13:89-91 